\title{
166. The Minimum Area of Convex Curves for Given Diameter and Perimeter
}

\author{
By Denzaburo Hemmi \\ Department of Mathematics, Yamagata University, Japan \\ (Comm. by Z. Suetuna, M.J.A., Nov. 12, 1954)
}

$\S 1$. Among the quantities concerning ovals the following are of fundamental importance: the area $F$, the perimeter $L$, the diameter $D$ and the thickness $\Delta$. Various relations between these quantities have been investigated by Kubota and others. However some of them, so-called minimum problems of a certain kind, remain unsolved. Already we, Kubota and I, solved two problems ${ }^{1)}$ of them as follows:

$$
\begin{aligned}
& F \geqq 3 \Delta\left\{\sqrt{D^{2}-\Delta^{2}}+\Delta\left(\sin ^{-1} \frac{\Delta}{D}-\frac{\pi}{3}\right)\right\}-\frac{\sqrt{3}}{2} D^{2} \text { for } D \geqq \Delta \geqq \sqrt{3} D / 2, \\
& 2 F \geqq \Delta L-\sqrt{3} \Delta^{2} \sec ^{2} \theta \quad \text { for } \pi \Delta \leqq L \leqq 2 \sqrt{3} \Delta,
\end{aligned}
$$

where $\theta$ is the root of $\tan \theta-\theta=(L-\pi \Delta) /(6 \Delta)$ in the interval $0 \leqq \theta \leqq \pi / 6$.

Before printing our results similar studies ${ }^{23)}$ were published in Germany and U.S.A. About that time Prof. T. Kubota died who often gave me kind advices and was my joint worker. The publication of our paper was delayed as we could not solve the $(L, D)$ problem: the problem of the minimum figures for $F$ when $D$ and $L$ are so given that $3 D<L<\pi D$. In the two papers above-mentioned, the former did not refer to the $(L, D)$ problem and the latter, M. Sholander's paper, gave the partial results for this problem and concluded as follows: "It is now natural to conjecture that the minimum figure is a triarc $R S T$ in the form of polygon inscribed in the Reuleaux triangle $R S T$. Assuming the truth of conjecture, a much more accurate description of the figure can be given. It remains doubtful, however, whether for $3 D<L<\pi D$ a simple inequality giving lower bounds for $F$ in terms of $L$ and $D$ exists which is better than Kubota's inequality". On the other hand I had

1) T. Kubota and D. Hemmi: Some problems of minima concerning ovals, J. Math. Soc. Japan, which was read at the annual meeting of the Math. Soc. of Japan held in June 2, 1951.

2) D. Ohmann: Extremalprobleme für konvexe Bereiche der euklidischen Ebene, Math. Z., 55, 347-352 (1952).

3) M. Sholander: On certain minimum problems in the theory of convex curves, Trans. Amer. Math. Soc., 73, 139-173 (1952). 
already got a lower bound for $F$ better than Kubota's inequality in the following form: ${ }^{4)}$

$2 F \geqq D^{2}\{[\pi / 2 \theta] \sin 2 \theta+\sin (2 \theta[\pi / 2 \theta])-\sqrt{3}\} \quad\left(\geqq D L \cos \theta-\sqrt{3} D^{2}\right)$, where [ ] is Gauss' notation and $\theta$ is the root of $[\pi / 2 \theta] \sin \theta+\cos$ $([\pi / 2 \theta] \theta)=L / 2 D$ in the interval $0 \leqq \theta \leqq \pi / 6$. But this inequality is not sharp enough, for the equality does not occur unless $L=6 n D$ sin $(\pi / 6 n), n=1,2,3, \ldots$.

Recently I solved the $(L, D)$ problem; and I shall summarize the result in this note.

\$2. If the polar tangential equation of an oval $\&$ is given by $p=p(\theta)$, the central oval $p=p(\theta)+p(\theta+\pi)$ is called the breadth curve of $\mathfrak{E}$. Let $\mathfrak{B}_{0}$ be a breadth curve of $\mathfrak{E}$ whose centre is $O$ and $P_{1} P_{2} P_{3} P_{4} P_{5} P_{6}$ be a convex polygon inscribed in $\mathfrak{B}_{0}$. When the polygon is symmetric with respect to $O$ and $P_{2} P_{3}=\frac{1}{2} P_{1} P_{4}$, then $P_{1} P_{2} P_{3} P_{4} P_{5} P_{6}$ will be called the base hexagon.

Let $\mathfrak{B}_{i}(i=1,2)$ be the translation of $\mathfrak{B}_{0}$ by $\overrightarrow{O P}_{i}(i=1,2)$, then the convex hull of the part common to three convex domains with boundary $\mathfrak{B}_{0}, \mathfrak{B}_{1}$ and $\mathfrak{B}_{2}$ will be called the asymmetric oval determined by $P_{1} P_{2} P_{3} P_{4} P_{5} P_{6}$ and $\mathfrak{B}_{0}$. The asymmetric oval is the same oval called "allgemeines Reuleauxdreieck" by Ohmann or triarc by Sholander.

The asymmetric oval is called "to be flat" if the breadth curve has at least one pair of rectilinear parts which coincide with sides of a base hexagon. The flatness of ovals was discussed by Sholander first.

The oval is called "to be $(L, D)$-deformable" if it is deformable into another oval which has the same perimeter and diameter and less area. By Blaschke's selection theorem, we see there exists a solution of the $(L, D)$ problem and therefore we get the solution by eliminating all $(L, D)$-deformable ovals. From a lemma ${ }^{5)}$ on oval we see that the solution of the $(L, D)$ problem is a non-asymmetric oval. Furthermore Sholander proved: No polygon is a solution of the $(L, D)$ problem. Thus we can state that no non-asymmetric polygon is a solution of the $(L, D)$ problem. Moreover we can prove that no flat polygon is a solution of the $(L, D)$ problem when $3 D<L<\pi D$. Besides any asymmetric polygon is $(L, D)$-deformable into one of the following three kinds of ovals;

4) D. Hemmi: On the minimum area of closed convex domains with an assigned breadth, Bull. Yamagata Univ., 2, 157-170 (1953).

5) Let $\mathfrak{B}$ be a breadth curve and $\mathfrak{S}$ be a hexagon of maximal area among all base hexagons inscribed in $\mathfrak{B}$, then the asymmetric oval determined by $\subseteq$ and $\mathfrak{B}$ is of minimum area among all ovals with the breadth assigned by $\mathfrak{B}$. Loc. cit. 1). Similar but not the same proofs are found in 2) 3), other two proofs in 4). 
1. asymmetric polygon, non-flat, whose base hexagon is a regular hexagon of sides $D$,

2. flat polygon,

3. non-asymmetric polygon.

Thus we get a theorem which implies Sholander's conjecture: when $3 D<L<\pi D$, solutions of the $(L, D)$ problem are non-flat asymmetric polygons, whose base hexagons are regular of sides $D . .^{6)}$

Furthermore, by Favard's result, we see our problem can be formulated analytically as follows:

$$
\begin{aligned}
& \frac{D^{2}}{2}\left\{\left[\frac{\pi}{6 x}\right] \sin 2 x+\sin 2\left(\frac{\pi}{6}-x\left[\frac{\pi}{6 x}\right]\right)+\left[\frac{\pi}{6 y}\right] \sin 2 y+\sin 2\left(\frac{\pi}{6}-y\left[\frac{\pi}{6 y}\right]\right)\right. \\
& \left.\quad+\left[\frac{\pi}{6 z}\right] \sin 2 z+\sin 2\left(\frac{\pi}{6}-z\left[\frac{\pi}{6 z}\right]\right)-2 \sin \frac{\pi}{3}\right\}=\text { minimum }
\end{aligned}
$$

under the conditions

$$
\begin{aligned}
2 D & \left\{\left[\frac{\pi}{6 x}\right] \sin x+\sin \left(\frac{\pi}{6}-x\left[\frac{\pi}{6 x}\right]\right)+\left[\frac{\pi}{6 y}\right] \sin y+\sin \left(\frac{\pi}{6}-y\left[\frac{\pi}{6 y}\right]\right)\right. \\
& \left.+\left[\frac{\pi}{6 z}\right] \sin z+\sin \left(\frac{\pi}{6}-z\left[\frac{\pi}{6 z}\right]\right)\right\}=L, \\
0 & <x<\frac{\pi}{6}, \quad 0<y<\frac{\pi}{6} \text { and } 0<z<\frac{\pi}{6},
\end{aligned}
$$

where [ $]$ is Gauss' notation and the constants $D$ and $L$ are given so that $3 D<L<\pi D$.

Suppose $z$ be fixed. After some elementary calculations for maxima and minima, we see the necessary conditions for the minimum problem (1) may be expressible as follows:

(I) in the case when both $\pi / 6 x$ and $\pi / 6 y$ are not integers,

$$
\cos x+\cos \left(\frac{\pi}{6}-x\left[\frac{\pi}{6 x}\right]\right)=\cos y+\cos \left(\frac{\pi}{6}-y\left[\frac{\pi}{6 y}\right]\right)
$$

and

$$
\frac{\sin x-\left[\frac{\pi}{6 x}\right] \sin \left(\frac{\pi}{6}-x\left[\frac{\pi}{6 x}\right]\right)}{\left[\frac{\pi}{6 x}\right]\left\{\cos \left(\frac{\pi}{6}-x\left[\frac{\pi}{6 x}\right]\right)-\cos x\right\}}+\frac{\sin y-\left[\frac{\pi}{6 y}\right] \sin \left(\frac{\pi}{6}-y\left[\frac{\pi}{6 y}\right]\right)}{\left[\frac{\pi}{6 y}\right]\left\{\cos \left(\frac{\pi}{6}-y\left[\frac{\pi}{6 y}\right]\right)-\cos y\right\}} \geqq 0 ;
$$

(II) in the case when one of $\pi / 6 x$ and $\pi / 6 y$ is not integer, for example, $\pi / 6 x \neq$ integer and $\pi / 6 y=$ integer,

$$
2 \cos y \leqq \cos x+\cos \left(\frac{\pi}{6}-x\left[\frac{\pi}{6 x}\right]\right) \leqq 1+\cos y ;
$$

(III) in the case when both $\pi / 6 x$ and $\pi / 6 y$ are integer,

6) D. Hemmi: On a minimum problem in the theory of ovals, Bull. Yamagata Univ., 3, 1-11 (1953). 


$$
1+\cos x-2 \cos y \geqq 0
$$

and

$$
1+\cos y-2 \cos x \geqq 0 .
$$

The cases (II) and (III) are minimal conditions for angular points of the function $F^{\prime}(x)$ defined by the parametric equations

$$
\begin{aligned}
& F=\left[\frac{\pi}{6 \theta}\right] \sin 2 \theta+\sin 2\left(\frac{\pi}{6}-\theta\left[\frac{\pi}{6 \theta}\right]\right), \\
& x=\left[\frac{\pi}{6 \theta}\right] \sin \theta+\sin \left(\frac{\pi}{6}-\theta\left[\frac{\pi}{6 \theta}\right]\right) .
\end{aligned}
$$

One of the difficulties of our problem is that the function $F(x)$ has many convex parts, concave parts and angular points which handled together and this gives rise to the existence of numerous minimal points. Besides the variation is so small that the evaluation seems hard, and if $L$ approaches $\pi D$, then extreme values become much more complicated. I pass through these difficulties by the following lemmas. ${ }^{\text {) }}$

We denote by $a_{n}$, for any positive integer $n$, the value $\pi /(6 n)$, by $b_{n}$, for $n \geqq 2$, the root of $\sin x=n \sin \left(a_{1}-n x\right)$ in the interval $\left(a_{n+1}, a_{n}\right)$, by $c_{n}$, for any integer $n \geqq 3$, the root of $\cos x+\cos$ $\left(a_{1}-n x\right)=1+\cos a_{n}$ in the interval $\left(a_{n+1}, b_{n}\right)$ and by $c^{*}$ the root of $\cos x+\cos \left(a_{1}-2 x\right)=2 \cos a_{3}$ in the interval $\left(b_{2}, a_{2}\right)$.

Lemma 1. Let $n \geqq 2, \cos x+\cos \left(a_{1}-n x\right)=\cos y+\cos \left(a_{1}-n y\right)$ where $x$ and $y$ satisfy $c_{n}<x<b_{n}<y<a_{n}$ for $n \geqq 3$ or $a_{3}<x<b_{2}<y<c^{*}$ for $n=2$. Then there holds

$$
\frac{\sin x-n \sin \left(a_{1}-n x\right)}{\cos \left(a_{1}-n x\right)-\cos x}+\frac{\sin y-n \sin \left(a_{1}-n y\right)}{\cos \left(a_{1}-n y\right)-\cos y}<0 .
$$

Lemma 2. Let $n \geqq 3,(n-1)(\alpha-\beta) \leqq \beta<n(\alpha-\beta)$ and $0<\alpha<a_{1}$. Then there exists $\bar{\alpha}$ such that $n \sin \alpha+(n+1) \sin \beta=n \sin (n+1) \beta / n+n$ $\sin \bar{\alpha}+\sin n(\alpha-\bar{\alpha})$ and that $n \sin 2 \alpha+(n+1) \sin 2 \beta>n \sin 2(n+1) \beta / n+n$ $\sin 2 \bar{\alpha}+\sin 2 n(\alpha-\bar{\alpha})$ in the interval $n \alpha /(n+1)<\bar{\alpha}<\alpha$.

Lemma 3. Let $m$ be 1 or $2, n$ be an integer $\geqq 2$ and $f(x), g(y)$ and $h(y)$ be functions as follows:

$$
\begin{aligned}
& f(x)=(m+1)\left\{n \sin x+\sin \left(a_{1}-n x\right)\right\} \quad \text { for } \quad b_{n} \leqq x \leqq a_{n} \\
& g(y)= \begin{cases}m n \sin a_{n}+n \sin y+\sin \left(a_{1}-n y\right) & \text { for } a_{n+1} \leqq y \leqq c_{n} \\
m\left\{n \sin y+\sin \left(a_{1}-n y\right)\right\}+(n+1) \sin a_{n+1} & \text { for } b_{n} \leqq y \leqq a_{n}\end{cases} \\
& h(y)=\left\{\begin{array}{l}
m n \sin 2 a_{n}+n \sin 2 y+\sin 2\left(a_{1}-n y\right) \quad \text { for } a_{n+1} \leqq y \leqq c_{n} \\
m\left\{n \sin 2 y+\sin 2\left(a_{1}-n y\right)\right\}+(n+1) \sin 2 a_{n+1} \text { for } b_{n} \leqq y \leqq a_{n}
\end{array}\right.
\end{aligned}
$$

where the first branches of $g(y)$ and $h(y)$ in the interval $a_{n+1} \leqq y \leqq c_{n}$ are to be considered only in the case $n \geqq 3$ and vanish in the case

7) D. Hemmi: The minimum area of convex curves for given perimeter and diameter, Bull. Yamagata Univ., 3, 55-76 (1954). 
No. 9] The Minimum Area of Convex Curves for Given Diameter and Perimeter 795

$n=2$. Then the simultaneous equations $f(x)=g(y), f(2 x)=h(y)$ have a pair of solution $x=x_{0}, y=y_{0}$. If $f(x)=g(y)$ and $f(x)$ is less (greater) than $f\left(x_{0}\right)$, then $f(2 x)$ is less (greater) than $h(y)$.

Lemma 4. In Lemma 3, $y_{0}$ satisfies either $a_{n+1}<y_{0}<c_{n}$ for $n \geqq 4$, or $b_{n}<y_{0}<a_{n}$ for $n=2,3$.

By these lemmas, the $(L, D)$ problem is now solved for the case $3 D<L<\pi D$. The result can be divided in the three cases:

$$
\begin{aligned}
& \text { the case } 3 D<L \leqq 12 D \sin \frac{\pi}{12}, \\
& \text { the case } 6 n D \sin \frac{\pi}{6 n}<L \leqq 6(n+1) D \sin \frac{\pi}{6(n+1)}, n=2 \text { or } 3, \\
& \text { the case } 6 n D \sin \frac{\pi}{6 n}<L \leqq 6(n+1) D \sin \frac{\pi}{6(n+1)}, n \text { is an }
\end{aligned}
$$

integer $\geqq 4$. Further, denoting by $\alpha_{n, m}, \beta_{n, m}$ and $k_{n, m}$ the value of $x_{0}, y_{0}$ and $f\left(x_{0}\right)$ in Lemma 3 respectively, the case (2) is subdivided in

$$
\begin{aligned}
& 6 n D \sin \frac{\pi}{6 n}<L \leqq 2 D k_{n, 2}, \\
& 2 D k_{n, 2}<L \leqq 2(n+1) D \sin \frac{\pi}{6(n+1)}+2 D k_{n, 1}, \\
& 2(n+1) D \sin \frac{\pi}{6(n+1)}+2 D k_{n, 1}<L \leqq 6(n+1) D \sin \frac{\pi}{6(n+1)},
\end{aligned}
$$

and the case (3) is subdivided in

$$
\begin{aligned}
& 6 n D \sin \frac{\pi}{6 n}<L \leqq 2 D k_{n, 2}, \\
& 2 D k_{n, 2}<L \leqq 2 D\left\{2 n \sin \frac{\pi}{6 n}+(n+1) \sin \frac{\pi}{6(n+1)}\right\} \\
& 2 D\left\{6 n \sin \frac{\pi}{6 n}+(n+1) \sin \frac{\pi}{6(n+1)}\right\}<L \leqq 2 D\left\{n \sin \frac{\pi}{6 n}+k_{n, 1}\right\} \\
& 2 D\left\{n \sin \frac{\pi}{6 n}+k_{n, 1}\right\}<L \leqq 2 D\left\{n \sin \frac{\pi}{6 n}+2(n+1) \sin \frac{\pi}{6(n+1)}\right\}, \\
& 2 D\left\{n \sin \frac{\pi}{6 n}+2(n+1) \sin \frac{\pi}{6(n+1)}\right\}<L \leqq 6 D(n+1) \sin \frac{\pi}{6(n+1)} .
\end{aligned}
$$

In the following the inequality giving minimum $F$ in each case can be expressed as (i), in which $\theta$ is determined by the equation (ii).

The case (1).

$$
\begin{aligned}
& 2 F \geqq 3 D^{2}\left\{\sin 2 \theta+\sin 2\left(\frac{\pi}{6}-\theta\right)\right\}-\sqrt{3} D^{2} ; \\
& L=6 D\left\{\sin \theta+\sin \left(\frac{\pi}{6}-\theta\right)\right\} ; \quad a_{1}>\theta \geqq a_{2} .
\end{aligned}
$$

The cases $(2,1)$ and $(3,1)$.

$$
2 F \geqq 3 D^{2}\left\{n \sin 2 \theta+\sin 2\left(\frac{\pi}{6}-n \theta\right)\right\}-\sqrt{3} D^{2} ;
$$


(ii)

$$
L=6 D\left\{n \sin \theta+\sin \left(\frac{\pi}{6}-n \theta\right)\right\} ; \quad a_{n}>\theta \geqq \alpha_{n, 2} .
$$

The case $(2,2)$.

$$
\begin{aligned}
2 F \geqq 2 D^{2}\{n \sin 2 \theta & \left.+\sin 2\left(\frac{\pi}{6}-n \theta\right)\right\} \\
& +D^{2}(n+1) \sin \frac{\pi}{3(n+1)}-\sqrt{3} D^{2} ;
\end{aligned}
$$

$$
L=4 D\left\{n \sin \theta+\sin \left(\frac{\pi}{6}-n \theta\right)\right\}+2 D(n+1) \sin \frac{\pi}{6(n+1)} ;
$$

The case $(2,3)$.

$$
\beta_{n, 2}>\theta \geqq \alpha_{n, 1} \text {. }
$$

(i) $2 F \geqq D^{2}\left\{n \sin 2 \theta+\sin 2\left(\frac{\pi}{6}-n \theta\right)\right\}+2(n+1) D^{2} \sin \frac{\pi}{3(n+1)}-\sqrt{3} D^{2}$;

(ii ) $L=2 D\left\{n \sin \theta+\sin \left(\frac{\pi}{6}-n \theta\right)\right\}+4(n+1) D \sin \frac{\pi}{6(n+1)}$;

The case $(3,2)$.

$$
\beta_{n, 1}>\theta \geqq a_{n+1} \text {. }
$$

(i ) $\quad 2 F^{\prime} \geqq D^{2}\left\{n \sin 2 \theta+\sin 2\left(\frac{\pi}{6}-n \theta\right)\right\}+2 n D^{2} \sin \frac{\pi}{3 n}-\sqrt{3} D^{2}$;

(ii) $\quad L=2 D\left\{n \sin \theta+\sin \left(\frac{\pi}{6}-n \theta\right)\right\}+4 n D \sin \frac{\pi}{6 n}$;

The case $(3,3)$.

$$
\beta_{n, 2}>\theta \geqq a_{n+1} \text {. }
$$

(i) $2 F \geqq 2 D^{2}\left\{n \sin 2 \theta+\sin 2\left(\frac{\pi}{6}-n \theta\right)\right\}+(n+1) D^{2} \sin \frac{\pi}{3(n+1)}-\sqrt{3 D^{2}}$;

(ii) $L=4 D\left\{n \sin \theta+\sin \left(\frac{\pi}{6}-n \theta\right)\right\}+2(n+1) D \sin \frac{\pi}{6(n+1)}$;

The case $(3,4)$.

$$
a_{n}>\theta \geqq \alpha_{n, 1} \text {. }
$$

$$
\begin{aligned}
2 F \geqq D^{2}\{n \sin 2 \theta & \left.+\sin 2\left(\frac{\pi}{6}-n \theta\right)\right\} \\
& +n D^{2} \sin \frac{\pi}{3 n}+(n+1) D^{2} \sin \frac{\pi}{3(n+1)}-\sqrt{3} D^{2} ;
\end{aligned}
$$

$$
\begin{gathered}
L=2 D\left\{n \sin \theta+\sin \left(\frac{\pi}{6}-n \theta\right)\right\} \\
+2 n D \sin \frac{\pi}{6 n}+2(n+1) D \sin \frac{\pi}{6(n+1)} ; \\
\quad \beta_{n, 1}>\theta \geqq a_{n+1} .
\end{gathered}
$$

The case $(3,5)$.

(i) $\quad 2 F \geqq D^{2}\left\{n \sin 2 \theta+\sin 2\left(\frac{\pi}{6}-n \theta\right)\right\}$

(ii)

$$
+2(n+1) D^{2} \sin \frac{\pi}{3(n+1)}-\sqrt{3} D^{2}
$$

$$
L=2 D\left\{n \sin \theta+\sin \left(\frac{\pi}{6}-n \theta\right)\right\}+4(n+1) D \sin \frac{\pi}{6(n+1)} ;
$$

$$
a_{n}>\theta \geqq a_{n+1} \text {. }
$$

